\title{
Direct White Light Emission from Carbon Nanodots (C-dots) in solution processed Light Emitting Diodes.
}

\begin{abstract}
Sofia Paulo-Mirasol, ${ }^{a, b}$ Eugenia Martin
We describe the preparation of inverted white light emitting diodes by solution processing. The active layer is formed uniquely by Carbon Nanodots (C-dots) that display white-light emission at voltage close to $5 \mathrm{~V}$ when combined with metal oxides as charge transport layers. Moreover, we have demonstrated that the white light is not the product of charge transfer between the polymer selective contact and the C-dots but the result of the different recombination processes within the $\mathrm{C}$-dots.
\end{abstract}

\section{Introduction}

Over the past 15 years, new colloidal quantum dots technologies have burst in both the academic and the industrial field $^{1,2}$. Quantum dots are defined as nanometer scale semiconductor crystals with a physical size smaller than the radius of the Bohr exciton, that, consequently, show quantum confinement. This means that, in general, the bandgap changes according to their size and thus their optical properties. In addition, the shape can be tuned to rods, nanowires or dots using different synthetic strategies. Due to their interesting optical properties, the nanocrystals have been used in optoelectronic devices like quantum dots light emitting diodes (QLEDs) that have emerged during the last years as the new LEDs generation due to better color definition over the entire visible spectrum ${ }^{3,4,5}$. When compared to organic semiconductors, quantum dots present broader absorption, narrower emission peaks and higher color purity.

In a simplistic view, the structure of the QLEDs consist of a sandwiched layer of emissive quantum dots between electrodes and selective contact layers. When a voltage bias is applied the holes and the electrons, called carriers, recombine radiatively in the emissive layer.

The most studied quantum dots are those composed of III-V elements such as GaP, InP or GaN and II-VI elements such as ZnS, CdTe, CdSe whose size ranges between 1-10nm. Lately, lead perovskite nanocrystals are also being intensively studied due their optoelectronic properties similar to cadmium based quantum dots such as high quantum yield and narrow emission, among others ${ }^{6,7}$. As far as we know, the best results are obtained using cadmium based or perovskite-like nanocrystals as emissive layer, with a quantum yield higher than $80 \% 8,9,10,11$. Notwithstanding the properties of these materials, they do contain toxic elements such as cadmium, lead, or selenium.
Thus, a challenge remains to replace these harmful elements for others with null or lower toxicity.

A new generation of quantum dots based on carbon nanostructures (C-dots) has recently emerged. Their low toxicity, environment-friendly and biocompatibility is a good alternative to heavy metal-containing nanomaterials. In addition, carbon based materials can be prepared using biocompatible products such as sugar or citric acid and, moreover, can be synthesized in non-halogenated solvents like ethanol or water ${ }^{12}$. The efforts of the research community have resulted in a multitude of examples of devices containing C-dots ranging from bio-imaging ${ }^{13,14}$, sensors ${ }^{15}$, solar cells ${ }^{16,17}$ or light emitting diodes ${ }^{18}$. However, this approach has still some limitations such as their low emission quantum yield and the color emission tuning. For instance, Wang in 2011 and Zhang in 2013, used 1-hexadecylamine (HDA) as passivating ligand for the synthesis of C-Dots and the construction of white emitting LEDs based on C-Dots, reporting luminance values below 100 $\mathrm{cd} / \mathrm{m}^{219,20}$. Despite the efforts to improve the quantum yield, it remains lower compared to the cadmium-based quantum dots 21,22. The reasons for this low luminance are assigned to aggregation induced quenching effects and poor film formation of the active layer ${ }^{23}$. In addition, the origin of the photoluminescence emission band of C-Dots is still under study $^{24,25}$. More successful approaches have been reported when using the C-Dots as white light converters in combination with UV-LED chips as excitation sources ${ }^{26,27}$.

In this work, we describe the use of C-Dots as single emitter in the emissive layer in the fabrication of solution processed inverted light emitting diodes. In these type of devices, we combine the robustness given by the metal oxides selective contact in the electron and hole transport layer with the emissive properties of the C-Dots. The device is based on a multi-stack structure using ZnO nanoparticles (NPs) as electron transport layer and Poly (9-vinylcarbazole) (PVK) as hole transport layer in substitution of the widely used hygroscopic PEDOT:PSS (Figure 1). Molybdenum oxide is deposited on top of the PVK to favor ohmic contact with the metallic electrode. Our procedure allowed the synthesis of nanocrystals with broad electroluminescence suitable for white light emission LEDs with the advantage of using only one component in the emissive layer, facilitating its fabrication and avoiding degradation of diverse components at different rates. In general, the most common routes to obtain white light are: (i) by mixing red, green and blue or blue and yellow quantum $\operatorname{dots}^{8}$, though the efficiency could be lower due to reabsorption of emitted colors, or (ii) dispersing the mono colored emissive C-dots in polymeric 
host matrices such as CBP or PVK, that also contribute to light emission although the polymer: nanocrystal ratio is crucial to obtain white colour ${ }^{18,28,24}$. To the best of our knowledge, this is the first example of inverted QLEDs using C-Dots as single emitter and component in the active layer.
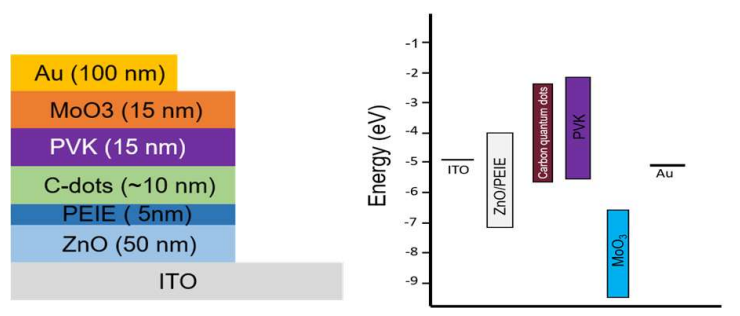

Figure 1. Description of the structure of the QDLEDs containing C-Dots and the energy levels of each component.

\section{Experimental}

\section{Materials and chemicals}

All materials and chemicals were used as received and without further purification: Citric acid (Fluka (C), 1-hexadecylamine (Sigma-Aldrich (C), 1-octadecene (Sigma-Aldrich (C), hexane (Sigma-Aldrich (C), Couramine 102, zinc acetate dihydrate (Sigma-Aldrich (C), potassium hydroxide (Sigma-Aldrich C), 2-[2(2-Methoxyethoxy)ethoxy]acetic acid (MEA, Sigma- Aldrich (C), Polyethylenimine, $80 \%$ ethoxylated solution $37 \mathrm{wt}$. \% in $\mathrm{H}_{2} \mathrm{O}$ (PEIE, Sigma-Aldrich (C), 2-methoxyethanol (97\%, SigmaAldrich()), methanol (Sigma-Aldrich $@)$, poly(N-vinyl carbazole (PVK, Sigma-Aldrich @), Chlorobenzene (Sigma-Aldrich (C).

\section{Synthesis of C-Dots}

The synthesis of C-Dots ( Figure 2) was carried out according to the method reported by Fu Wang et al. ${ }^{29}$. In brief, $15 \mathrm{~mL}$ of 1 octadecene (ODE) and $1.5 \mathrm{~g}$ of 1-hexadecylamine (HDA) were stirred in a three-neck flask under inert conditions during $30 \mathrm{~min}$ at room temperature. Then, the solution was heated up to 300 ${ }^{\circ} \mathrm{C}, 1 \mathrm{~g}$ of citric acid was quickly added and the solution was stirred during $3 \mathrm{~h}$. Once the solution was cooled down at room temperature, we added acetone to precipitate the C-dots and centrifuged at $4500 \mathrm{rpm}$ during $20 \mathrm{~min}$. Afterwards, the supernatant was separated and the solid was washed three times with hexane.

Figure 2. Schematic representation of the C-Dots synthesis.

\section{Characterization techniques}

The UV-Visible and fluorescence spectra were measured, using a $1 \mathrm{~cm}$ path length quartz cell in a Shimadzu(C) UV-Visible spectrophotometer 1700 and a Fluorolog@ Horiba Jobin Ivon spectrophotometer. The Time-correlated Single Photon
Counting (TCSPC) measurements were recorded in Edinburgh Instruments $@$ LifeSpec II spectrometer. The TEM images were recorded using a JEOL 1011 microscope. The cyclic voltammetry (CV) was performed with an Autolab (C) PGSTAT 30 electrochemical analyser at room temperature in acetonitrile solvent, using a platinum wire as counter electrode, a commercial $\mathrm{Ag} / \mathrm{AgCl}$ reference electrode and a platinum disc as working electrode. A solution of $0.1 \mathrm{M}$ tetrabutyl ammonium hexafluorophosphate $\left(\mathrm{TBAPF}_{6}\right)$ was used as supporting electrolyte. The sample was stirred and purged with nitrogen before the measurement. The scan rate was $0.01 \mathrm{Vs}^{-1}$.

\section{Fabrication of the inverted devices}

All the devices were fabricated using ITO-coated glasses (Indium doped Tin Oxide) (Xinyan Technology $\subseteq$, resistance $10 \Omega /$ square, $15 \times 15 \mathrm{~mm}$ ). The patterned ITO was cleaned with acetone for $15 \mathrm{~min}, 2$-propanol for $15 \mathrm{~min}$ in an ultrasound bath followed by UV/ozone treatment for $20 \mathrm{~min}$.

The synthesis of ZnO NPS was carried out using the procedure described in the bibliography ${ }^{30}$. ZnO NPs $(80 \mathrm{mg} / \mathrm{ml}$ in methanol) were deposited by spin coating at $5000 \mathrm{rpm}, 60 \mathrm{~s}$ and heated up for $30 \mathrm{~min}$ at $150{ }^{\circ} \mathrm{C}$ on top of the ITO. The polyethylenimine ethoxylated (PEIE 0,2\%wt 2-methoxyethanol) was spin coated on top of the ZnO NPs at $6000 \mathrm{rpm} 60 \mathrm{~s}$ and annealed at $110^{\circ} \mathrm{C}$ for $30 \mathrm{~min}$. After that, the solution of carbon quantum dots in hexane with different concentrations was spin coated at $1000 \mathrm{rpm} 60 \mathrm{~s}$ and baked at $100{ }^{\circ} \mathrm{C}$ for $10 \mathrm{~min}$. The Poly(N-vinyl carbazole (PVK) $10 \mathrm{mg} / \mathrm{ml}$ in chlorobenzene was deposited on top of the C-dots at $1000 \mathrm{rpm} 60 \mathrm{~s}$ and annealed at $110^{\circ} \mathrm{C} 10 \mathrm{~min}$. The fabrication procedure was done in air and all the solutions were filtered through a PTFE $0.2 \mu \mathrm{m}$ filter. Finally, thermal evaporation of $15 \mathrm{~nm}$ of $\mathrm{MoO}_{3}$ and $100 \mathrm{~nm}$ of Au was done at $10^{-6} \mathrm{mbar}$. The final area of the devices is $9 \mathrm{~mm}^{2}$.

\section{Results and discussion}

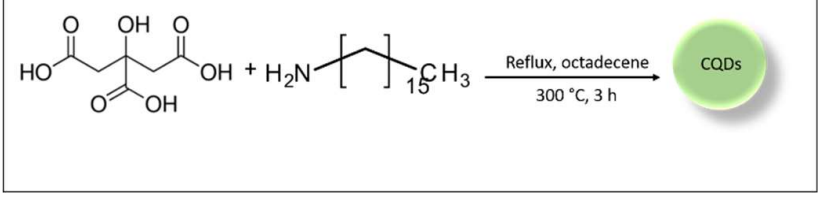


The size and shape of the C-dots were analysed by transmission electron microscopy (TEM, see supporting information, Figure S1) at different magnification scales. As illustrated, the C-dots present a spherical shape and are well dispersed without traces of molecular aggregates. In average, the C-dots size is $6 \pm 1.9$ $\mathrm{nm}$. Surface analysis was done using Fourier Transform Infrared spectroscopy (FT-IR). As can be seen in Figure S2, the presence of the stretching bands corresponding to the $\mathrm{C}=\mathrm{C}$ and $\mathrm{C}=\mathrm{N}$ in the region of $1500-1600 \mathrm{~cm}^{-1}$ confirms the anchoring of the HDA to the surface.

The optical characterization was carried out by using UV-Visible and photoluminescence spectroscopy in hexane (Figure 3 ). The C-Dots display strong excitonic absorption bands at $\lambda=230 \mathrm{~nm}$ and $\lambda=365 \mathrm{~nm}$ in the UV-Visible (Figure $3 \mathrm{top}$ ) and a minor peak at $490 \mathrm{~nm}$. These two first bands are attributed to two different photophysical processes. The first band is due to $\pi \rightarrow \pi^{*}$ transition of the $\mathrm{C}=\mathrm{C}$ bonds whereas the second is assigned to the $n \rightarrow \pi^{*}$ transition due to the amino groups of the surface passivating ligands ${ }^{31,25}$. The photoluminescence emission spectra (Figure 3 bottom) is excitation dependent displaying maximum emission bands between $\lambda=496 \mathrm{~nm}$ and $\lambda=548 \mathrm{~nm}$. Data obtained after excitation at $340 \mathrm{~nm}$ shows maximum emission at $460 \mathrm{~nm}$ with a full width at half maximum (FWHM) of $125 \mathrm{~nm}$ and PLQY of $44 \%$.

As mentioned above, the origin of the photoluminescence mechanism in C-Dots is still under discussion. Among the different hypothesis, there are two that have more scientific consensus: (i) trap assisted emission, independently of the location of the exciton ${ }^{32,33}$ and (ii) different emission states depending on the location of the exciton at the core of the $\mathrm{C}$ Dots or at their surface 25,18 . From our data, we assign the emission at different $\lambda$ to the electronic transitions at the $\mathrm{C}=\mathrm{O}$ groups, that will occur mainly at the core of the C-dots and the electronic transition at the $\mathrm{C}=\mathrm{N}$ groups, which will take place at the surface of the C-Dots where the surface passivation ligands are covalently attached ${ }^{34,35}$.

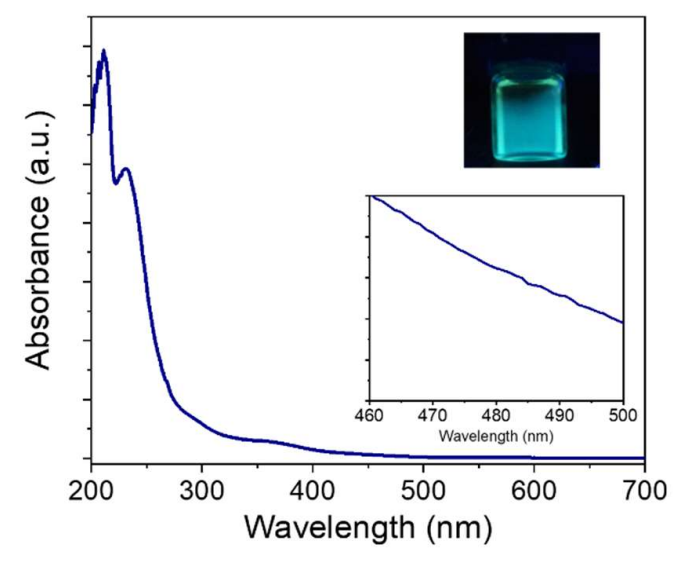

Figure 3. Top) the UV-Visible absorption spectra in hexane $10 \mathrm{mg} / \mathrm{ml}$; and bottom) the fluorescence emission spectra in hexane $10 \mathrm{mg} / \mathrm{ml}$ at room temperature obtained after excitation at different wavelengths. Insets in Figure 3 to between 460 and $500 \mathrm{~nm}$ to show the peak at $490 \mathrm{~nm}$.

Moreover, the C-Dots fluorescence decay lifetime was measured in solution at different wavelengths (Figure S5). The emission decays were fitted using a bi-exponential function (Equation 1).

$\tau(t)=A_{1} e^{-\left(\frac{t}{\tau 1}\right)}+A_{2} e^{-\left(\frac{t}{\tau 2}\right)} \quad$ Equation 1

where $A 1$ and $A 2$ are the amplitude of the radiative decay lifetime and $\tau$ represent the lifetime values. The results are shown in Table 1. The fitting lifetimes can be assigned to two different processes, being the fastest the recombination of the

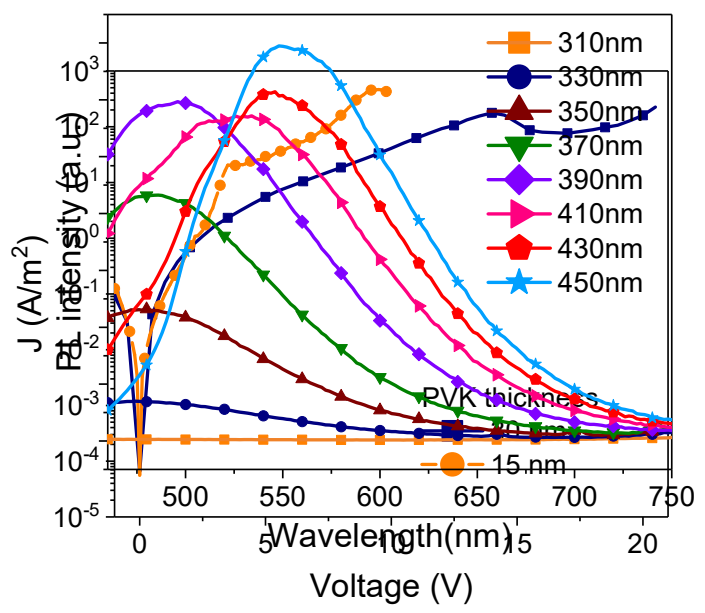

carriers at C-Dots surface and the slowest to the carrier recombination at the C-Dots core in good agreement with previous publications ${ }^{36}$. It is worth noticing that the energy of the excitation wavelength is related to the lifetime of the decays, since longer lifetimes are observed when the excitation energy is higher.

\begin{tabular}{|c|c|c|c|c|c|c|c|c|c|c|c|c|}
\hline$\lambda_{\text {exc }}$ & \multicolumn{4}{|c|}{$270 \mathrm{~nm}$} & \multicolumn{4}{c|}{$405 \mathrm{~nm}$} & \multicolumn{4}{c|}{$470 \mathrm{~nm}$} \\
\hline & $\begin{array}{c}\tau 1 \\
(\mathrm{~ns})\end{array}$ & $\begin{array}{c}\mathrm{A} 1 \\
(\%)\end{array}$ & $\begin{array}{c}\tau 2 \\
(\mathrm{~ns})\end{array}$ & $\begin{array}{c}\text { A2 } \\
(\%)\end{array}$ & $\begin{array}{c}\tau 1 \\
(\mathrm{~ns})\end{array}$ & $\begin{array}{c}\mathrm{A} 1 \\
(\%)\end{array}$ & $\begin{array}{c}\tau 2 \\
(\mathrm{~ns})\end{array}$ & $\begin{array}{c}\mathrm{A} 2 \\
(\%)\end{array}$ & $\begin{array}{c}\tau 1 \\
(\mathrm{~ns})\end{array}$ & $\begin{array}{c}\mathrm{A} 1 \\
(\%)\end{array}$ & $\begin{array}{c}\tau 2 \\
(\mathrm{~ns})\end{array}$ & $\begin{array}{c}\mathrm{A} 2 \\
(\%)\end{array}$ \\
\hline CDs & 4.8 & 93.8 & 19.5 & 6.2 & 3.8 & 99.5 & 14.8 & 0.5 & 2.5 & 96.7 & 10.3 & 3.3 \\
\hline
\end{tabular}

Table 1. Fluorescence decay of C-Dots measured in hexane $[5 \mathrm{mg} / \mathrm{mL}]$ after excitation at $\lambda=270 \mathrm{~nm}, 405 \mathrm{~nm}$ and $470 \mathrm{~nm}$ at room temperature.

The electrochemical properties of the $\mathrm{C}$-dots in solution have been measured by cyclic voltammetry using Ferrocene/Ferrocene ${ }^{+}$as internal standard indicating the presence of an irreversible oxidation wave at $1.6 \mathrm{~V}$. Using the value for the $0-0$ energy transition (EOO)-calculated from the UV-Visible and the fluorescence emission intersection point (Figure S4)-and the electrochemical data (Figure S3) we have estimated the Valence Band (VB) and Conduction Band (CB) energies for the $\mathrm{C}$-Dots to be $5.66 \mathrm{eV}$ and $3.13 \mathrm{eV}$, respectively. 
These values results appropriated for the preparation of QLEDs using $\mathrm{ZnO}$ and the combination of the polymer $\mathrm{PVK}$ and $\mathrm{MoO}_{3}$ as electron and hole selective contacts, respectively. Moreover, an interlayer of PEIE (polyethylenimine ethoxylated) between the $\mathrm{ZnO}$ and the C-Dots was used too. The PEIE is used to inject more efficiently the carriers ${ }^{37}$ and helps to create a uniform surface for the subsequent deposition of C-Dots ${ }^{38}$.

All layer thicknesses were adjusted to optimize the device luminance efficiency. For instance, Figure 4 illustrates the differences in the PL properties for different concentration of $\mathrm{C}$ Dots deposited by spin coating. As can be seen, there is an inverse correlation between the concentration of C-Dots and the photoluminescence. We can explain this by considering selfquenching processes depending on the concentration of the $\mathrm{C}$ Dots, in agreement with previous observations by other authors? ${ }^{7}$.

Figure 4. The photoluminescence spectra for different $\mathrm{C}$-dots concentrations deposited on glass when excited at $\lambda$ exc $=360 \mathrm{~nm}$ at room temperature.

Once the concentration of C-Dots is optimised, we prepared complete devices by solution processing under air conditions. The quality of the layer of C-Dots onto ZnO/PEIE has been checked by AFM, observing the presence of aggregates that do

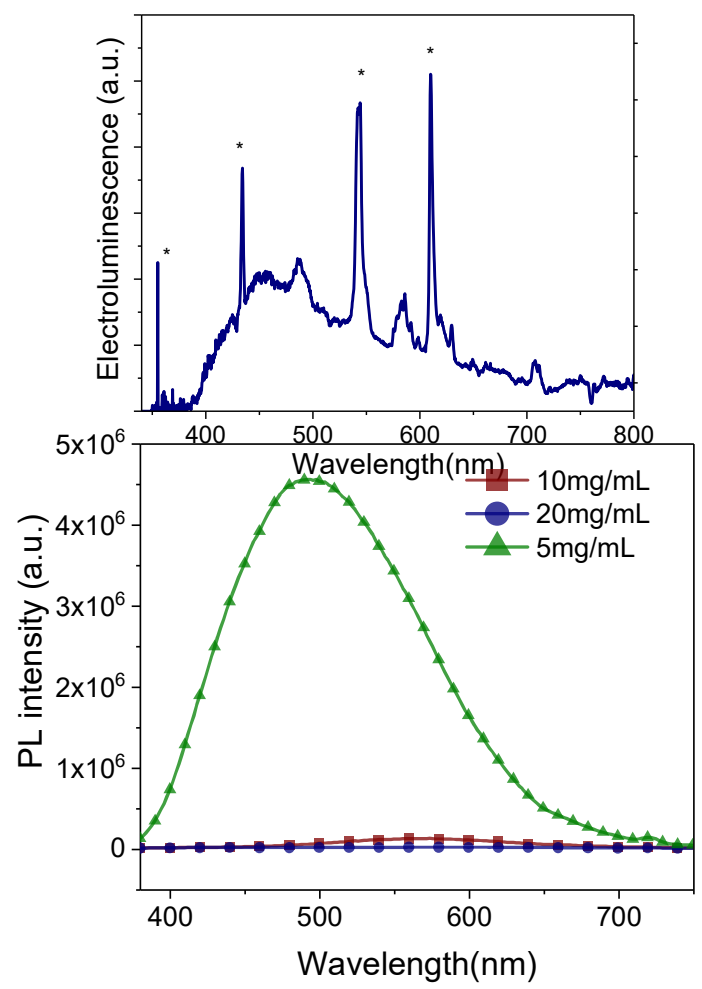

not fully cover the layer underneath (Figure S6). The deposition of PVK onto the C-Dots results in a homogeneous layer.

Other parameters such as the optimal thickness for the PVK layer has also been tested. Figure 5 shows the current density (Figure 5 top) and luminance versus applied voltage (Figure 5 bottom) for devices prepared modifying the thickness of PVK.
The device in operation display some inhomogeneity that are assigned to the low uniformity of the C-Dot layer. As can be seen

\begin{tabular}{|c|c|c|c|}
\hline PVK & $\begin{array}{c}\text { Turn-on voltage } \\
V^{\text {on }}(\mathrm{V})\end{array}$ & $\begin{array}{c}\text { Max. Luminance } \\
\left(\mathrm{Cd} / \mathrm{m}^{2}\right)^{\mathrm{a}}\end{array}$ & $\begin{array}{c}\text { Current Efficiency } \\
(\mathrm{Cd} / \mathrm{A})\end{array}$ \\
\hline $30 \mathrm{~nm}$ & 10.5 & $7(6.5)$ & 0.07 \\
\hline $15 \mathrm{~nm}$ & 5 & $21(17)$ & 0.06 \\
\hline
\end{tabular}

in Table 2, the turn-on voltage decreased from $10 \mathrm{~V}$ to $5 \mathrm{~V}$ just decreasing the thickness of PVK. This decrease optimizes the charge balance into the active layer and thus, increases current density and radiative recombination. Moreover, the obtained current efficiency values are higher than those reported previously in quantum dot LEDs containing C-Dots as single emitter.

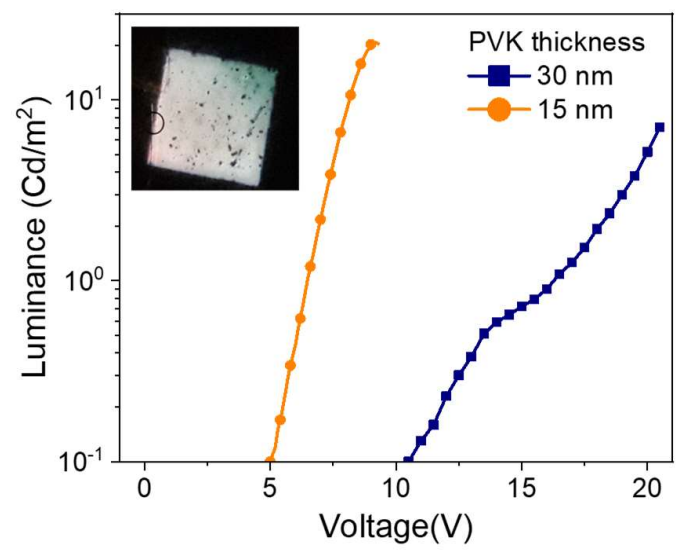

Figure 5. (top) Current density versus applied voltage as a function of the PVK thickness for complete devices; (bottom) luminance versus applied voltage as a white light emission at $6 \mathrm{~V}$. The concentration of C-Dots was $5 \mathrm{mg} / \mathrm{mL}$.

${ }^{\text {a }}$ average luminance of C-dots LEDs reported in round brackets.

Table 2. Summary of the device performances. The $V^{\text {on }}$ is the turn-on voltage at 0.1 $\mathrm{cd} / \mathrm{m}^{2}$.

Figure 6 top shows the electroluminescence spectra of the CDots LEDs at $8 \mathrm{~V}$. We would like to notice that the highlighted peaks correspond to noise present during the measurement. The electroluminescence emission encompasses a broad range of wavelengths that resulted in white light emission in agreement with the broad PL signal observed in solution and film. No contribution from the PVK is observed. This is of utmost importance as, often to obtain while light electroluminescence, it is necessary to combine materials with different emission wavelengths. In our C-Dots LEDs the broad emission spectra suffices to obtain white light at voltages as low as $8 \mathrm{~V}$. Figure 6 bottom shows the $\mathrm{CIE}$ color coordinates of $(0.284,0291)$, which are close to the pure white light coordinates of $(0.33,0.33)$. 
Figure 6. The electroluminescence of the C-dots LEDs at $8 \mathrm{~V}$ measured at room temperature (top). CIE color coordinates of QLEDs with C-dots as single emitte (bottom).

The origin of the white color using a single emitter is still under debate. For example, Wang and collaborators reported that white light emission was associated with energy transfer among various emitting centers in the C-Dots, corresponding to different energy transitions ${ }^{19}$. On the other hand, Zhang et al. assigned the variation of the color emission to the presence of different mechanisms of recombination on the C-Dots when used as single emitter ${ }^{20}$. Taking into account our observations from the fluorescence decays, we believe that there are two radiative photoluminescence mechanisms in the C-Dots involving different energies: one originating in the core with longer lifetime (see Table 1) and a second process that is faster and originates in the surface of C-Dots. Thus, we conclude that the variation of the current injection controls the activation of the two radiative process that happen inside the $\mathrm{C}$-dot. The combined emission from different energy states results in white light emission at an adequate current injection rate.

Regarding the stability of the devices, three different diodes were measured every two minutes to check their lifetime and the light emission quality. The successive measurement rounds resulted in an increase of the turn-on voltages and lower luminance values. Remarkably, the current density variation

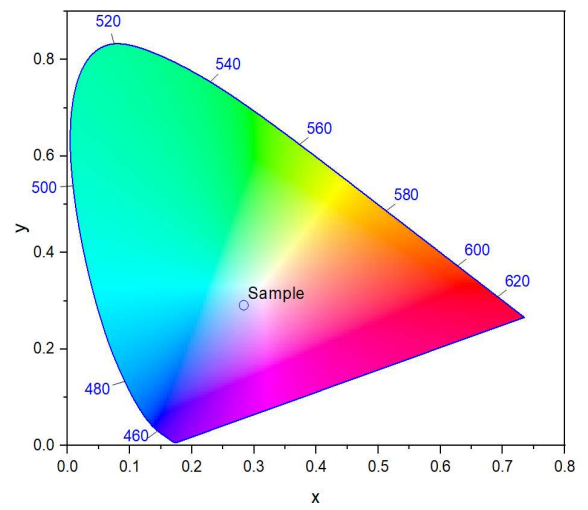

with the applied bias did not change. After three rounds,

measurements were stopped and the devices stored under air. Then 24 hours later, the cycles began but no light emission could be detected. We assign the rapid decrease of luminance and the increment of the turn on voltage with the saturation of the traps where the electronic transition that result in light emission take place. Since the current density does not change, we do not believe that degradation of the active layer took place since there is not an increase of the resistance. However, there is an increase in the turn on voltage indicating that higher energy is required for light emission. Our hypothesis that current injection controls light emission would support the idea that higher energy is required to overcome the filling of the deeper traps in order to observe luminance.

Finally, and despite the presence of an intermixed photoactive layer originating from the heterogeneous C-Dots layer, to confirm that the electroluminescence was originated in the CDots layer and not from the PVK, we fabricated a LED without C-Dots. Figure 7 shows the performance of inverted devices with the structure glass/ITO/ZnO:PEIE/PVK/ $/ \mathrm{MoO}_{3} / \mathrm{Au}$. We have deposited $30 \mathrm{~nm}$ of PVK to be able to compare between both types of LEDs. It can be seen that, in comparison to C-Dots containing devices, the turn on voltage increases while the maximum luminance decreases whereas, the current density values are lower indicating unbalanced charge injection. In addition, as shown in the inset of Figure 7 , the emitted light is purple. The second inset in Figure 7 shows the PL spectrum of the PVK only device exhibiting a maximum at $380 \mathrm{~nm}$ after excitation at $340 \mathrm{~nm}$. This maximum cannot be appreciated in the EL spectrum of the C-dots LED device, thus confirming that the origin of the white light emission is due to the C-dot layer.

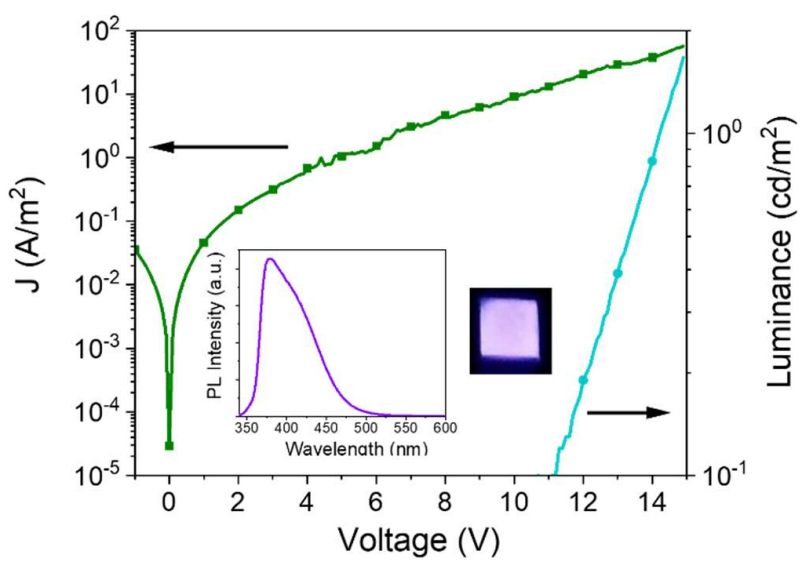

Figure 7. Current density (blue line) and luminance (green line) versus applied voltage of the LED made without C-Dots. The insets show the digital picture of the spectra of the device after excitation at $340 \mathrm{~nm}$.

\section{Conclusions}

In summary, we have shown a versatile pathway for the synthesis of carbon dots (C-Dots) using a bottom up process with citric acid as carbon source and 1-hexadecylamine as capping ligand. The characterization of the optical and 
electrochemical characteristics indicate that the C-dots are appropriated to be used as electroactive thin layer in inverted solution processed light emitting diodes. Their quantum yield, and the conduction and valence bands are suitable for the use of $\mathrm{ZnO}$ as electron selective contact and $\mathrm{PVK}$ and $\mathrm{MoO}_{3}$ as hole transport material in complete devices.

Importantly, the use of C-Dots as single emitter in light emitting devices gives rise to white light emission at room temperature without the need of encapsulation with higher efficiency values than precedent examples. The fabrication of the device has been carried out by solution processing (spin coating) under air conditions, pointing out the potential of the C-Dots for upscaling in roll-to-roll devices. Regarding the performance, the broad emission peaks allows to obtain white light without the need of mixing different materials, which is a great advantage as simplifies further the device multilayer structure.

We acknowledge that further optimization of the device is required to achieve values close to their non-carbon QDs counterparts but, taking into account the lack of toxic elements, the green chemistry procedures for their synthesis and the easy-to-make device fabrication we are already working further on this direction using C-dots as carrier recombination layers in light emitting devices.

\section{Conflicts of interest}

No potential conflict of interest is reported by the authors

\section{Notes and references}

‡ Supplementary Information: TEM images, FTIR spectrum, fluorescence decays and electrochemical characterization of Cdots.

$1 \quad$ X. Liang, S. Bai, X. Wang, X. Dai, F. Gao, B. Sun, Z. Ning, Z. Ye and Y. Jin, Chem. Soc. Rev., 2017, 46, 1730-1759.

2 X. Dai, Y. Deng, X. Peng and Y. Jin, Adv. Mater., 2017, 29, 1607022.

3 J. Albero, J. N. Clifford and E. Palomares, Coord. Chem. Rev., 2014, 263-264, 53-64.

4 A. P. Litvin, I. V Martynenko, F. Purcell-Milton, A. V Baranov, A. V Fedorov and Y. K. Gun'ko, J. Mater. Chem. A, 2017, 5, 13252-13275.

5 Y. Jiang, S.-Y. Cho and M. Shim, J. Mater. Chem. C, 2018, 6, 2618-2634.

6 B. Han, B. Cai, Q. Shan, J. Song, J. Li, F. Zhang, J. Chen, T. Fang, Q. Ji, X. Xu and H. Zeng, Adv. Funct. Mater., 2018, 28, 1804285.

7 Y. Ling, Y. Tian, X. Wang, J. C. Wang, J. M. Knox, F. PerezOrive, Y. Du, L. Tan, K. Hanson, B. Ma and H. Gao, Adv. Mater., 2016, 28, 8983-8989.

8 B. C. Hames, I. Mora-Seró and R. S. Sánchez, Nano Res., 2018, 11, 1575-1588.

9 L. Zhang, X. Yang, Q. Jiang, P. Wang, Z. Yin, X. Zhang, H. Tan, Y. Yang, M. Wei, B. R. Sutherland, E. H. Sargent and J.

You, Nat. Commun., 2017, 8, 15640.

10

11

12

13

14

15

16

17

18

19

20

21
Q. Lin, L. Wang, Z. Li, H. Shen, L. Guo, Y. Kuang, H. Wang and L. S. Li, ACS Photonics, 2018, 5, 939-946.

Y. Shang and Z. Ning, Natl. Sci. Rev., 2017, 4, 170-183.

T. T. Meiling, P. J. Cywiński and I. Bald, Sci. Rep., 2016, 6, 28557.

H. Liu, L. Zhang, M. Yan and J. Yu, J. Mater. Chem. B, 2017, 5, 6437-6450.

F. R. Baptista, S. A. Belhout, S. Giordani and S. J. Quinn, Chem. Soc. Rev., 2015, 44, 4433-4453.

F. Li, X.-H. Yu, F.-Y. Kong, Z.-X. Wang and W. Wang, RSC Adv., 2017, 7, 31281-31286.

S. Paulo, E. Palomares and E. Martinez-Ferrero, Nanomaterials, 2016, 6, 157.

S. Paulo, G. Stoica, W. Cambarau, E. Martinez-Ferrero and E. Palomares, Synth. Met., 2016, 222, 17-22.

J. Xu, Y. Miao, J. Zheng, H. Wang, Y. Yang and X. Liu, Nanoscale, 2018, 10, 11211-11221.

F. Wang, Y. Chen, C. Liu and D. Ma, Chem. Commun., 2011, 47, 3502-3504.

X. Y. Zhang, Y. Zhang, Y. Wang, S. Kalytchuk, S. V Kershaw, Y. H. Wang, P. Wang, T. Q. Zhang, Y. Zhao, H. Z. Zhang, T. Cui, Y. D. Wang, J. Zhao, W. W. Yu and A. L. Rogach, ACS Nano, 2013, 7, 11234-11241.

K.-H. Lee, J.-H. Lee, H.-D. Kang, B. Park, Y. Kwon, H. Ko, C. Lee, J. Lee and H. Yang, ACS Nano, 2014, 8, 4893-4901.

H. Zhang, H. Li, X. Sun and S. Chen, ACS Appl. Mater. Interfaces, 2016, 8, 5493-5498.

Y. Ding, Q. Chang, F. Xiu, Y. Chen, Z. Liu, C. Ban, S. Cheng, J. Liu and W. Huang, Nanoscale, 2018, 10, 4189-4193.

F. Yuan, Z. Wang, X. Li, Y. Li, Z. Tan, L. Fan and S. Yang, Adv. Mater., 2017, 29, 1604436.

S. Zhu, Y. Song, X. Zhao, J. Shao, J. Zhang and B. Yang, Nano Res., 2015, 8, 355-381.

F. Zhang, X. Feng, Y. Zhang, L. Yan, Y. Yang and X. Liu, Nanoscale, 2016, 8, 8618-8632.

X. T. Feng, F. Zhang, Y. L. Wang, Y. Zhang, Y. Z. Yang and X. G. Liu, Appl. Phys. Lett., 2015, 107, 1-6.

W. Kwon, Y.-H. Kim, C.-L. Lee, M. Lee, H. C. Choi, T.-W. Lee and S.-W. Rhee, Nano Lett., 2014, 14, 1306-1311.

F. Wang, S. Pang, L. Wang, Q. Li, M. Kreiter and C. Liu, Chem. Mater., 2010, 22, 4528-4530.

F. C. K. and Y. T. and R. T. and J. W. Andreasen, Nanotechnology, 2008, 19, 424013.

Z. Jiang, M. J. Krysmann, A. Kelarakis, P. Koutnik, P. Anzenbacher, P. J. Roland, R. Ellingson and L. Sun, MRS Adv., 2017, 2, 2927-2934.

T. Feng, Q. Zeng, S. Lu, X. Yan, J. Liu, S. Tao, M. Yang and B. Yang, ACS Photonics, 2018, 5, 502-510.

S. Hu, X. Meng, F. Tian, W. Yang, N. Li, C. Xue, J. Yang and Q. Chang, J. Mater. Chem. C, 2017, 5, 9849-9853.

D. Chen, H. Gao, X. Chen, G. Fang, S. Yuan and Y. Yuan, ACS Photonics, 2017, 4, 2352-2358.

H. Nie, M. Li, Q. Li, S. Liang, Y. Tan, L. Sheng, W. Shi and S. X.-A. Zhang, Chem. Mater., 2014, 26, 3104-3112.

Y. Ding, F. Zhang, J. Xu, Y. Miao, Y. Yang, X. Liu and B. Xu, RSC Adv., 2017, 7, 28754-28762.

D. I. Son, H. H. Kim, D. K. Hwang, S. Kwon and W. K. Choi, J. 
Mater. Chem. C, 2014, 2, 510-514.

H. H. Kim, S. Park, Y. Yi, D. I. Son, C. Park, D. K. Hwang and W. K. Choi, Sci. Rep., 2015, 5, 8968. 